\title{
Identification of Seed Purity on Hybrid Japonica Rice 'Huayou 14' Using SSR Markers
}

Yao Danqing ${ }^{1 *} \square$, Xia Jianming ${ }^{1}$, Lou Jianfeng ${ }^{1}$, Gu Qinqin ${ }^{1}$, Liu Jian ${ }^{1}$, Zhang Yu ${ }^{1}$, Zhang Qiuli ${ }^{1}$, Zhang Weiwei ${ }^{2}$

1 Shanghai Agricultural Technology Extension and Service Center, Shanghai, 201103, China

2 Shanghai Vocational College of Agriculture and Forestry, Shanghai, 201699, China

\Corresponding author email: ydq_726@163.com

Molecular Plant Breeding, 2021, Vol.12, No.25 doi: $10.5376 / \mathrm{mpb} .2021 .12 .0025$

Received: 19 Apr., 2021

Accepted: 25 Aug., 2021

Published: 03 Sep., 2021

Copyright $\odot 2021$ Yao et al., This article was first published in Molecular Plant Breeding in Chinese, and here was authorized to translate and publish the paper in English under the terms of Creative Commons Attribution License, which permits unrestricted use, distribution, and reproduction in any medium, provided the original work is properly cited.

Preferred citation for this article:

Yao D.Q., Xia J.M., Lou J.F., Gu Q.Q., Liu J., Zhang Y., Zhang Q.L., and Zhang W.W., 2021, Identification of seed purity on hybrid japonica rice 'Huayou 14' using SSR markers, Molecular Plant Breeding, 12(25): 1-7 (doi: $\underline{10.5376 / \mathrm{mpb} .2021 .12 .0025}$ )

\begin{abstract}
In this study, the hybrid japonica rice 'Huayou 14' and its parents were used as materials, using SSR molecular marker technology, to select primers and identify seed purity according to its polymorphism. From 48 pairs of candidate SSR molecular markers widely distributed on 12 rice chromosomes, 6 pairs of primers were screened, which showed stable codominance between the hybrid and its parents. Completely distinguished, using these primers to carry out molecular purity identification of 'Huayou 14' seeds, and comparing the identification results with the field planting purity identification results, the results showed that the identification results of the two were basically the same. Finally, the RM274 and RM1195 marker combinations with the most polymorphic sites and the most significant differences in specificity were finally determined as core primers, and a more rapid and effective technical method for identifying the seed purity of hybrid japonica rice 'Huayou 14' was established.
\end{abstract}

Keywords SSR marker; Hybrid japonica rice; 'Huayou 14'; Purity identification

Rice (Oraza sativa) is one of the most important food crops in the world, especially the hybrid rice varieties with excellent parental traits. Due to its high yield and high quality, rice has been widely planted and promoted in all parts of China, effectively ensuring the sustainable and stable agricultural production with high yield and high quality. In actual production, the purity of hybrid lines directly affects the yield and quality of crops, and the full play of heterosis potential must be based on high purity of hybrid lines, so the seed purity identification has always been the most important index in the promotion and application of improved varieties.

Field plot identification is the most widely used method to identify the purity of hybrid rice varieties (Sun et al., 2014). However, its identification cycle is long, the workload is large, the cost is high, and it is easy to be affected by the environment. Therefore, it is of great significance to explore a simple, fast, accurate and cheap method of hybrid seed purity identification for promoting the compliance operation of enterprises and improving the regulatory efficiency of the government on the seed market. In recent years, the rapid development of molecular marker technology has made it an important method to identify the purity of seeds (Yashitola et al., 2002). In particular, SSR marker is characterized by its simplicity, convenience, easy standardization, low requirements on the quantity and quality of DNA, accurate and reliable detection results, and good repeatability. It has been widely used in the purity identification of rapeseed (Me et al., 2010), rice (Wang et al., 2014), corn (Wu et al., 2012), cucumber (Cui et al., 2015) and other crop hybrids, and has been recommended by the International Association for Seed Inspection (ISAT) as the preferred method for variety purity and authenticity detection.

'Huayou 14' is a japonica hybrid rice with high quality and high yield bred in Shanghai in recent years. The average yield of Shanghai rice is increased by more than $10 \%$, and the main quality index reaches the national standard of high quality rice level one. The planting of $7 \times 10^{5} \mathrm{mu}$ has been promoted in the Yangtze River Delta region, and the economic and social benefits are remarkable. This study intends to use hybrid japonica rice 'Huayou 14' as the experimental material, and establish a more rapid and effective technical method to identify the seed purity of hybrid japonica rice 'Huayou 14' based on SSR marker technology, so as to provide technical support for large-scale promotion. 


\section{Results and Analysis}

\subsection{Genome-wide DNA extraction from rice 'Huayou 14' and its parents}

The results showed that there was only one band in the genomic DNA extracted from 'Huayou 14', its female parent 'Shen 9A' and its male parent 'Fan 14', as well as all the DNA of the samples to be tested. The results showed that the purity and concentration of the extracted genomic DNA met the requirements of subsequent PCR amplification (Figure 1).

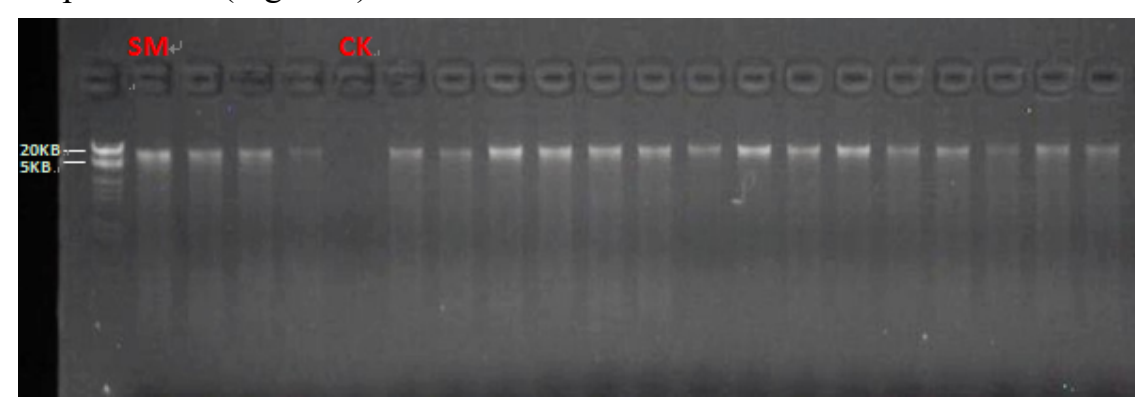

Figure 1 Electrophoretogram of rice genomic DNA

Note: The first hole was size maker; The sixth hole was negative control

\subsection{Screening and validation of 'Huayou 14' specific primers}

1.2.1 Establish DNA fingerprint data of 'Huayou 14' to screen specific primers

The experimental results showed that most of the SSR primers could amplify stable and clear bands, among which there were 15 pairs of SSR primers between 'Huayou 14' and its parents that could distinguish the hybrid from its parents (Table 1). As shown in the test results (Figure 2), the band type of 'Huayou 14' is complementary to the two parents, which can distinguish the parents from 'Huayou 14', preliminarily indicating that the 15 pairs of SSR primers can identify the purity of 'Huayou 14 '.

\subsubsection{Qualitative identification, screening and verification of specific primers by hybrid types}

Through SSR primer specificity analysis on hybrids, 15 pairs of SSR markers that could distinguish each hybrid type from $F_{1}$ generation seeds were finally screened (Figure 2; Figure 3). The results showed that the 15 pairs of SSR primers of parental complementary type were consistent with the 15 pairs of SSR primers screened by hybrid combination and its parent DNA fingerprint. These 15 pairs of markers can effectively identify all phenotypes of hybrids. In addition, the genotype of some hybrid samples of type 1-17 selected through field investigation of plant phenotypes was consistent with that of $F_{1}$ generation seeds of 'Huayou 14' (Sample 18) (Figure 3), indicating that molecular marker-assisted verification could more accurately identify hybrid types than morphological observation.

\subsection{Validation of the validity of SSR markers in the identification of variety purity}

Fifteen pairs of SSR molecular markers were selected to verify the validity of SSR marker identification method for two consecutive years, and six primers were selected to be used to identify the purity of varieties (Figure 4; Figure 5). In the first year, 264 single seeds of 'Huayou 14' were identified for molecular purity in sample No. 2017108.The results showed that taking A05 as an example, there were 260 grains per plant with fully complementary specific bands with their parents, and the purity of 'Huayou 14' was $98.5 \%$ by calculation. Among the other false 'Huayou 14', there were 0 specific banding seeds of 'Huayou 14' and 4 seeds of 'Shen9A'. According to field identification statistics, 17 of the 1000 hybrid plants were inconsistent with the morphology of 'Huayou 14', that is, the seed purity of 'Huayou 14' was 98.3\%.In the second year, the number is no. 2018120 samples of 196 grains' flower optimal 14 single molecular purity identification of seed, the above method, respectively, the two samples of the field investigation and appraisal and molecular marker detection results are compared (Table 2), the result was consistent, show that selected primers' 14 'optimal seed purity identification results are correct and credible. Artificial emasculation hybridization was carried out during the whole process of reproduction, so the false seeds with the same band type as the parent may be from the mixed female seeds. 
Table 1 'Huayou 14' hybrid and its parental primer screening results

\begin{tabular}{|c|c|c|c|c|c|}
\hline Number & Primer & Chromosomes & Primer set & Annealing temperature $\left({ }^{\circ} \mathrm{C}\right)$ & Primer sequence $\left(5^{\prime} \rightarrow 3^{\prime}\right)$ \\
\hline A01 & RM583 & 1 & I & 55 & $\begin{array}{l}\text { 5'Primer: agatccatccetgtggagag } \\
\text { 3'Primer: gcgaactcgcgttgtaatc }\end{array}$ \\
\hline A05 & RM274 & 5 & I & 55 & $\begin{array}{l}\text { 5'Primer: cctcgcttatgagagettcg } \\
\text { 3'Primer: cttctccatcactcccatgg }\end{array}$ \\
\hline A07 & RM336 & 7 & I & 55 & $\begin{array}{l}\text { 5'Primer: cttacagagaaacggcatcg } \\
\text { 3'Primer: gctggtttgtttcaggttcg }\end{array}$ \\
\hline A11 & RM209 & 11 & I & 55 & $\begin{array}{l}\text { 5'Primer: atatgagttgctgtcgtgeg } \\
\text { 3'Primer: caacttgcatcctccectcc }\end{array}$ \\
\hline A12 & RM19 & 12 & I & 55 & $\begin{array}{l}\text { 5'Primer: caaaaacagagcagatgac } \\
\text { 3'Primer: ctcaagatggacgccaaga }\end{array}$ \\
\hline B01 & RM1195 & 1 & II & 55 & $\begin{array}{l}\text { 5'Primer: atggaccacaaacgaccttc } \\
\text { 3'Primer: cgactccettgttcttctgg }\end{array}$ \\
\hline B03 & RM232 & 3 & II & 55 & $\begin{array}{l}\text { 5'Primer: ccggtatcettcgatattgc } \\
\text { 3'Primer: ccgacttttcetcctgacg }\end{array}$ \\
\hline B06 & RM253 & 6 & II & 55 & $\begin{array}{l}\text { 5'Primer: tccttcaagagtgcaaaacc } \\
\text { 3'Primer: gcattgtcatgtcgaagcc }\end{array}$ \\
\hline B10 & RM258 & 10 & II & 55 & $\begin{array}{l}\text { 5'Primer: tgctgtatgtagctcgcacc } \\
\text { 3'Primer: tggcctttaaagctgtcgc }\end{array}$ \\
\hline $\mathrm{C} 02$ & RM561 & 2 & III & 55 & $\begin{array}{l}\text { 5'Primer: gagctgttttggactacggc } \\
\text { 3'Primer: gagtagctttctcccaccc }\end{array}$ \\
\hline $\mathrm{C} 11$ & RM21 & 11 & III & 55 & $\begin{array}{l}\text { 5'Primer: acagtattccgtaggcacgg } \\
\text { 3'Primer: gctccatgagggtggtagag }\end{array}$ \\
\hline D02 & RM490 & 1 & IV & 55 & $\begin{array}{l}\text { 5'Primer: atctgcacactgcaaacacc } \\
\text { 3'Primer: agcaagcagtgctttcagag }\end{array}$ \\
\hline D04 & RM423 & 2 & IV & 55 & $\begin{array}{l}\text { 5'Primer: agcacccatgcettatgttg } \\
\text { 3'Primer: cctttttcagtagcectccc }\end{array}$ \\
\hline D09 & RM542 & 7 & IV & 55 & $\begin{array}{l}\text { 5'Primer: tgaatcaagcccctcactac } \\
\text { 3'Primer: ctgcaacgagtaaggcagag }\end{array}$ \\
\hline D12 & RM7102 & 12 & IV & 55 & $\begin{array}{l}\text { 5'Primer: taggagtgtttagagtgcca } \\
\text { 3'Primer: tcggtttgcttatacatcag }\end{array}$ \\
\hline
\end{tabular}

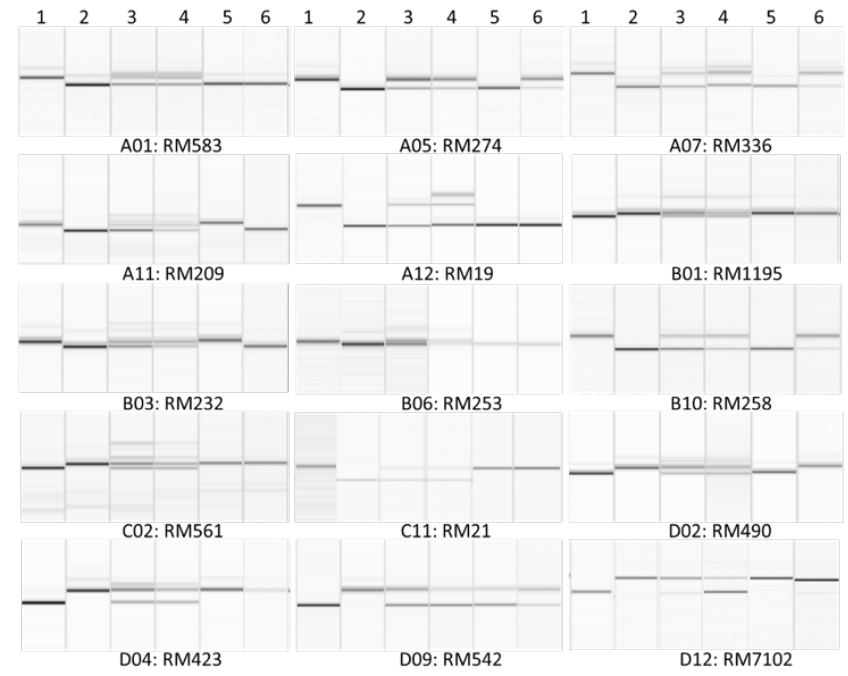

Figure 2 Results of primer specificity analysis of 'Huayou 14' hybrid and its parental Analysis of plant specificity in 2018120 plot planting identification

Note: 1: Male line; 2: Female line; 3: 'Huayou 14'; 4: Sample of hybrid in plot planting identification field in 2018120; 5, 6: Sample of miscellaneous plants in plot planting identification field in 2018120 

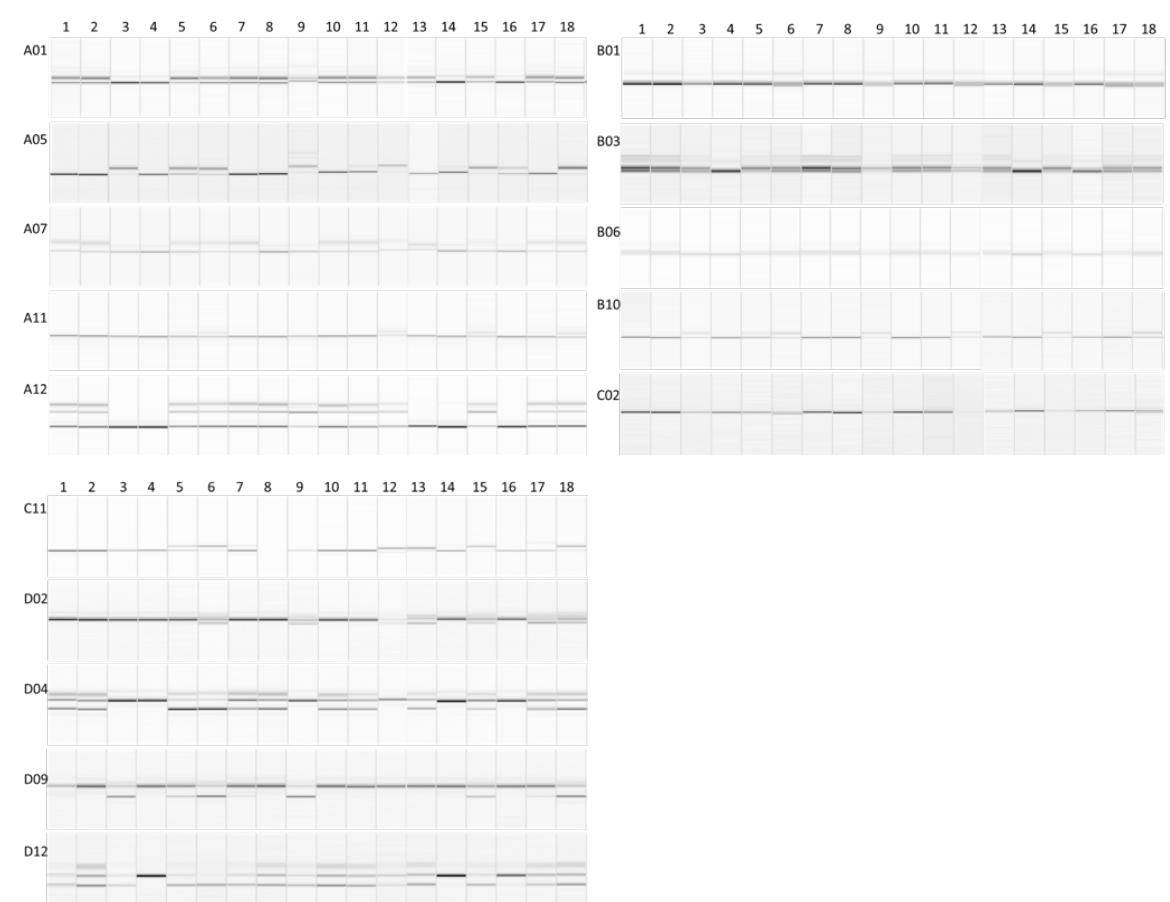

Figure 3 Analysis of plant specificity in 2017108 plot planting identification

Note: 1-17: Sample of miscellaneous plants in plot planting identification field in 2017108; 18: Sample of hybrid in plot planting identification field in 2017108

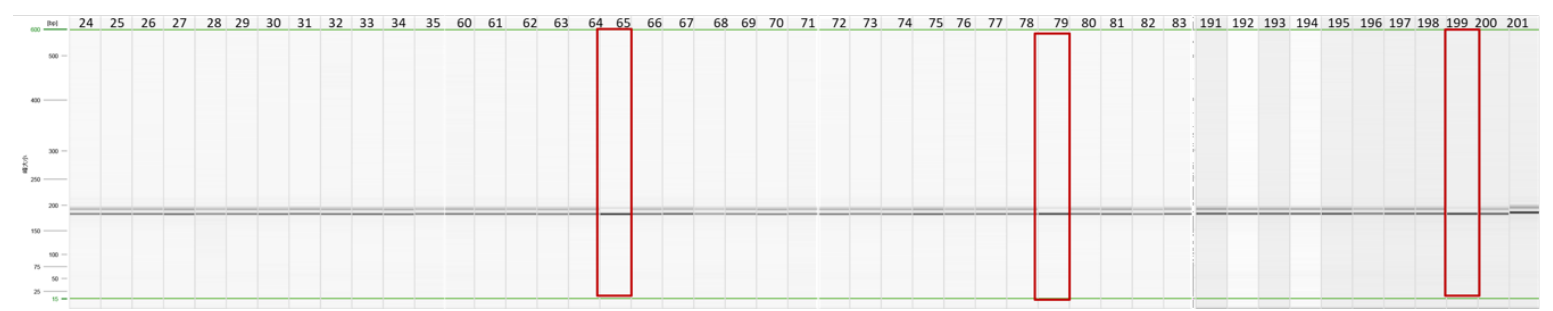

Figure 4 The results of primer Alin the group of 'Huayou 14'

Note: $65,79,199$ : The seeds were false, other of seeds were hybrids
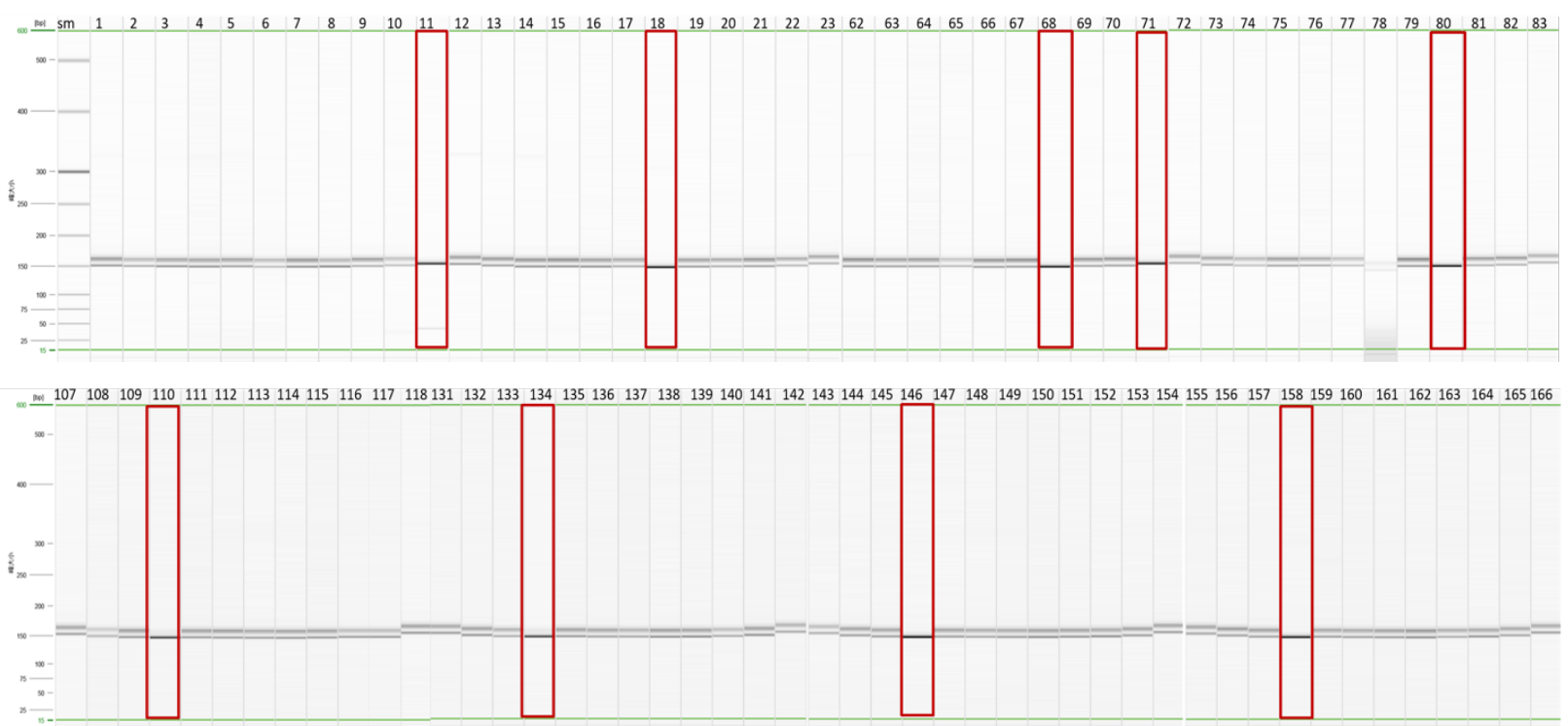

Figure 5 The results of primer A5in the group of 'Huayou 14'

Note: $11,18,68,71,80,110,134,146,158$ : The seeds were false, other of seeds were hybrids 
Molecular Plant Breeding 2021, Vol.12, No.25, 1-7

http://genbreedpublisher.com/index.php/mpb

Table 2 Comparison of field purity test and molecular purity test

\begin{tabular}{|c|c|c|c|c|c|c|c|c|c|}
\hline \multirow{2}{*}{$\begin{array}{l}\text { Sample } \\
-\end{array}$} & \multicolumn{3}{|c|}{ Field test } & \multicolumn{6}{|l|}{ Molecular test } \\
\hline & $\begin{array}{l}\text { Purity } \\
(\%)\end{array}$ & $\begin{array}{l}\text { Total } \\
\text { number }\end{array}$ & $\begin{array}{l}\text { Number of } \\
\text { hybrid plants }\end{array}$ & Primers & $\begin{array}{l}\text { Purity } \\
(\%)\end{array}$ & $\begin{array}{l}\text { Total } \\
\text { grains }\end{array}$ & $\begin{array}{l}\text { Specific } \\
\text { individuals }\end{array}$ & $\begin{array}{l}\text { Deviation } \\
(\%)\end{array}$ & $\begin{array}{l}\text { Specific individual } \\
\text { number }\end{array}$ \\
\hline \multirow[t]{6}{*}{2017108} & 98.3 & 1000 & 17 & A01: RM538 & 98.8 & 264 & 3 & 0.5 & $65,79,199$ \\
\hline & & & & A05: RM274 & 98.5 & 264 & 4 & 0.3 & $27,65,79,199$ \\
\hline & & & & A07: RM336 & 98.5 & 264 & 4 & 0.3 & $27,65,79,199$ \\
\hline & & & & B01: RM1195 & 98.5 & 264 & 4 & 0.3 & $27,65,79,199$ \\
\hline & & & & C02: RM561 & 98.5 & 264 & 4 & 0.3 & $27,65,79,199$ \\
\hline & & & & D02: RM490 & 98.5 & 264 & 4 & 0.3 & $27,65,79,199$ \\
\hline \multirow[t]{6}{*}{2018120} & 96.9 & 1000 & 31 & A01: RM538 & 95.9 & 196 & 8 & 1.0 & $\begin{array}{l}18,68,71,110,134,158, \\
165,169\end{array}$ \\
\hline & & & & A05: RM274 & 95.4 & 196 & 9 & 1.5 & $\begin{array}{l}11,18,68,71,80,110 \\
134,146,158\end{array}$ \\
\hline & & & & A07: RM336 & 96.9 & 196 & 6 & 0.0 & $\begin{array}{l}18,68,110,117,134, \\
158\end{array}$ \\
\hline & & & & B01: RM1195 & 94.4 & 196 & 11 & 2.5 & $\begin{array}{l}11,18,68,71,80,110 \\
134,146,158,165,169\end{array}$ \\
\hline & & & & C02: RM561 & 98.5 & 196 & 3 & 1.6 & $11,80,146$ \\
\hline & & & & D02: RM490 & 97.4 & 196 & 5 & 0.5 & $11,80,146,165,169$ \\
\hline
\end{tabular}

\section{Discussion}

The purity of hybrid rice seeds seriously affects its quality and yield. It is an important index to identify the quality of hybrid rice seeds. In the past, purity identification of hybrid rice varieties usually adopts the field plot planting authentication method, this method though the results more accurate, but the appraisal process to operating personnel's professional quality request is higher, need to have rich experience in production field, and authorized long cycle, high cost, low efficiency, now it is difficult to meet the requirements of marketization development of hybrid rice seeds. SSR molecular identification technology does not depend on the understanding of the characteristics of varieties, nor is it affected by planting, so the identification results are more reliable. At present, this technology has been widely used (Sun et al., 2014).

In this study, 48 pairs of SSR standard primers were used to analyze the genotype of hybrid japonica rice variety 'Huayou 14' and its parents (restorer line 'Fan 14' and sterile line 'Shen 9A'), and the DNA fingerprint data of the hybrid combination was established, and 15 pairs of specific markers were screened to distinguish 'Huayou 14' from its parents. At the same time, the hybrid plant types were investigated and identified in the field plot identification garden for two consecutive years. Through qualitative analysis of the hybrid plant types, 15 pairs of specific markers were screened and the results were consistent with the former, and the molecular identification method of seed purity of hybrid japonica rice 'Huayou 14' was preliminarily established. In order to verify the effectiveness of this method, six pairs of primers with significant specificity were selected to identify the molecular purity of hybrid japonica rice 'Huayou 14' seeds produced in 2017 and 2018, and the results were basically consistent with the field identification results. These results indicated that the identification of seed purity of hybrid japonica rice 'Huayou 14' by these 6 pairs of primers was accurate, simple and efficient. In addition, the six pairs of primers were used as candidate markers for the purity identification test of hybrid japonica rice seeds in other Yangtze River Delta regions, and found that the genetic polymorphism was high, which could also be used in the purity identification test of other varieties in the future.

Given that RM274 and RM1195 have the most polymorphic loci and the most significant difference in specificity, considering the cost of molecular identification, "molecular identification method for seed purity of hybrid japonica rice 'Huayou 14' based on RM274 and RM1195 marker combination" was finally determined. This technique is of great significance for the legal management of hybrid japonica rice variety 'Huayou 14' and the quality control of the government. 


\section{Materials and Methods}

\subsection{Experimental materials}

Standard samples of 'Huayou 14' and its parents (restorer line 'Fan 14' and sterile line 'Shen 9A') and $\mathrm{F}_{1}$ seeds of 'Huayou 14' produced in 2017 and 2018 (Sample No.: 2017108 and 2018120) were all provided by Shanghai Crop Seed Quality Testing Center. The primers were synthesized by Sangon Biotech (Shanghai) Co., Ltd.

A total of 1000 seeds were randomly selected from the samples numbered 2017108 and 2018120, respectively, and identified in the field plot in Hainan. The main hybrid types in the identified garden were analyzed for specificity, and SSR markers were screened out to distinguish the hybrid types.

\subsection{Extraction of rice genomic DNA}

'Huayou 14' and its parents (restorer line 'Fan 14' and sterile line 'Shen 9A'), as well as hybrid plant materials from the identification nursery, 20 seeds were taken from each sample and placed in a $2 \mathrm{~mL}$ circular bottom centrifuge tube, adding 2 steel balls and grinding with QIAGEN grinder at maximum frequency for 20 min to prepare DNA extraction. QIGAEN plant genome DNA kit was used for extraction. The DNA was extracted and detected by $0.7 \%$ agarose gel electrophoresis. The purity and concentration of DNA were measured by A NanoDrop2000c UV-vis spectrometer. The working solution was diluted to $40 \sim 50 \mathrm{ng} / \mu \mathrm{L}$ and prepared in the refrigerator at $4^{\circ} \mathrm{C}$.

The single seeds of rice samples 2017108 and 2018120 were used to verify the molecular purity identification method. The genomic DNA of rice was extracted by magnetic seed DNA beads with the GO-GPLF-FX96 kit produced by GeneOn BioTech Co., Ltd. The instruments included Thermo FLEX nucleic acid extraction instrument. Mix elf, centrifuge, metal bath/water bath/air bath.

\subsection{Primer screening and validation}

\subsubsection{Establish DNA fingerprint data of 'Huayou 14' to screen specific primers}

Using genomic DNA of 'Huayou 14' and its parents as a template, PCR amplification was performed to screen SSR primers with good polymorphism. Forty-eight pairs of SSR primers from the industrial standard "Technical Regulations for Rice Variety Identification --SSR Marker Method" issued by the ministry of Agriculture were selected for PCR amplification and genotype identification of rice varieties. The PCR amplification system was 20 $\mu \mathrm{L}$, and the amplification procedure was as follows: pre-denaturation at $94^{\circ} \mathrm{C}$ for $5 \mathrm{~min}$, denaturation at $94^{\circ} \mathrm{C}$ for $40 \mathrm{~s}$, annealing at $55^{\circ} \mathrm{C} \sim 60^{\circ} \mathrm{C}$ for $35 \mathrm{~s}$, extension at $72^{\circ} \mathrm{C}$ for $45 \mathrm{~s}$, a total of 37 cycles. The product was extended at $72^{\circ} \mathrm{C}$ for $10 \mathrm{~min}$ and stored at $4^{\circ} \mathrm{C}$. After amplification, capillary electrophoresis was used for analysis, and QIAxcelScreenGel version 1.4 software was used to analyze the original data to determine the genotype of each SSR locus. The band type of the hybrid was the complementary type of the two parents as the target primer.

\subsubsection{Qualitative identification, screening and verification of specific primers by hybrid types}

17 hybrid samples and 2 hybrid samples were selected in the field plot planting identification nursery (2017108 and 2018120) for two consecutive years. The hybrid samples were selected through morphological observation, and the specificity analysis of these hybrid strains was conducted with 48 pairs of SSR markers to further verify the effectiveness of screening primers. The total reaction system and the reaction procedure are as above.

\subsection{Verification of SSR markers for purity identification of varieties}

264 and 196 single seeds were randomly selected from samples 2017108 and 2018120, respectively. The selected population single 'Huayou 14' seeds were used to verify the screened SSR primers. The SSR primer screening test was the same as the above experiment, and the detection results of the screening primers on the population single seeds were observed and compared.

\subsection{Comparison of different purity identification methods for rice seeds}

In SSR molecular identification, SSR primers were used to identify randomly selected single seeds of 'Huayou 14'. The SSR primer test system and procedure were the same as the above process. In the conventional field plot identification, 1000 plants of 'Huayou 14' were planted in The Experimental base of Lingshui, Hainan, and their 
phenotypic traits were observed. Purity $(\%)=$ number of hybrid seeds/total number of plants $\times 100$. The similarity between SSR molecular identification method and field plot identification method was compared.

\section{Authors' contributions}

YDQ is the person in charge of the project, the experimental designer of this study and the executer of the experimental research. She completes the data analysis and writes the first draft of the paper. LJF, GQQ, ZY, ZQL provided the preparation of experimental materials for this study, ZWW, LJ participated in the design of the experiment and the analysis of the experimental results; XJM directed the design of the experiment and the writing and modification of the paper. All authors read and approved the final manuscript.

\section{Acknowledgments}

This research was supported by the Shanghai Agricultural System Youth Talent Development Program Fund (No. 9-1, 2018), the Whole industry chain of high quality rice green Production mode Development Project II (No. 1-1, 2018), and the research and demonstration of high quality rice green production technology (No. 3, 2018).

\section{References}

Cui X.H, Han Y.K., Du S.L., Wei A.M., Chen Z.W., and Liu N., 2015 SSR identification of seed purity of a New Cucumber Variety 'Jinyou 401', Zhongguo Guacai (Cucumis melo), (3): 38-39

Mei D.H., Li Y.C., Chen Y.F., Li Y.D., Xu Y.S., Hu Q., 2010, Identification of seed purity of Zhongyouza 12 by peroxidase isozyme and SSR markers, Nongye Jishu Xuebao (Journal of agricultural biotechnology), 18 (4): 815-821

Sun H.Y., Gu W.W., Wang S.Y., Xu G.M., Luo B., Yang Z.G., Shen Z.G., and Duanmu Y.X., 2014, Identification of seed purity on hybrid japonica rice 'Changyou 1' using SSR markers, Fenzi Zhiwu Yuzhong (Molecular Plant Breeding), 11(5): 557-561

Wang M.H., Zhang X.T., Wu G., Jiang Q., and Shi Y., 2019, DNA fingerprints construction and purity identification based on SSR markers for rice varieties in Ningbo city, Zhongguo Daomi (China Rice), 25(6): 50-54

Wang Y.F., Dong J.G., Dong Z.S., Qu L.Y., Ge J., Guo Y.F., HuY.M., and Lan G., 2011, Screening of SSR primers for identification the seed purity of variety qin10 in B. napus, Jiyinzuxue Yu Yingyong Shengwuxue (Genomics and Applied Biology), 30(1): 72-77

Wu Y.J., Xu J., and Che X.X., 2012, Application of SSR marker technique to purity testing of maize hybrid qiangsheng 16, Shanxi Nongye Kexue (Journal of Shanxi Agricultural Science), 40(6): 599-602

Yashitola J., Thirumumgan T., Sundaram R.M., Naseemllah M.K., Ramesha M.S., Sarma N.P., and Sonti Ramesh V., 2002, Assessment of purtity of fice hybrid using microsatellite and STS markers, Crop Sci., 42(4): 1369-1373

https://doi.org/10.2135/cropsci2002.1369 